\title{
CFD Analysis of Industrial Multi-Stage Impeller in Stirred Tank with Fractal Pattern Baffled and Impeller
}

\author{
MANSHOOR Bukhari, a, MDSAUFI Muhammad Faiq ${ }^{1, b}$, \\ ZAMAN Izzuddin ${ }^{1, c}$, and KHALID Amir ${ }^{1, d}$ \\ ${ }^{1}$ Centre for Energy and Industrial Environment Studies (CEIES), \\ Faculty of Mechanical and Manufacturing Engineering \\ Universiti Tun Hussein Onn Malaysia \\ 86400 Pt. Raja, Batu Pahat, Johor, MALAYSIA \\ abukhari@uthm.edu.my, bfaiqsaufi@gmail.com, 'izzuddin@uthm.edu.my, damirk@uthm.edu.my
}

Keywords: Stirred Tank, Fractal, Fractal Baffled

\begin{abstract}
This paper presents tool for analysis of CFD adapted for flows in multi-staged stirred vessels with fractal pattern baffled for industrial. In order to develop a good mixing process model for stirred tanks, several way have been investigated by using the computational fluid dynamic. Implementing fractal design into stirred tank's baffle and impeller are believed to influence the flow characteristic inside the stirred tank. The simulation of mixing process will be conducted by using multi-stage stirred tanks. Hence, the study is to simulate a fractal pattern baffled stirred vessels with fractal base of impeller. Four models with a new concept and different design of stirred tank have been introduced and studied. The multi-stages stirred tanks will equipped with fractal base pattern concept. The simulation is carry out by using the standard k- $\varepsilon$ turbulence model. The results will be analyze in order to compare the better method for the most effective in mixing. The flows produced in stirred tank are different and relevant with each model. The velocity profiles also give a relevant and quite impressive result by each model. At the end, the results will be examined and compared with each data that use a common type of baffle and impeller design.
\end{abstract}

\section{Introduction}

The progress of Computer Fluid Dynamics (CFD) for the last 20 years has made it an important tool for process mixing understanding in stirred tanks. Stirred tank reactor was commonly used in many industries such as chemical biotechnologies, chemical, food processing and many more [1]. Many design and specification were come out for stirred tanks and they were different according to its use [2]. Most of the study is to make the optimum stirred tank design configuration with good efficiencies of mixing process. Factors that influence the performance of mixing process are, mixing time, blade type, blade numbers, blade diameter and speed of the rotating blade and the use of baffle [3].

Efficiency here were measured on how fast the mixing completed their process while at the same time can maintain or even reduce the operation cost. According to the interest in designing the most effective stirred tank, many researches, was focused on the optimization of the design of the stirred tanks and impellers geometry. For example, Kchaou et al. [4] was compared an effect of the flat-blade turbine with $45^{\circ}$ and $-45^{\circ}$ pitched blade turbines on the hydrodynamic structure of the stirred tank. For the the effects of different inclined angle of impellers, Driss et al. [5] developed a computational study of the pitched blade turbines design effect on the stirred tank flow characteristics with inclined angle of $45^{\circ}, 60^{\circ}$ and $75^{\circ}$, on the local and global flow characteristics. Besides that, Mishra et al. [6] were investigated the flow occurring between dual Rushton impellers and the instantaneous velocity gradients distribution in the outlet of impeller blade using Laser Doppler Anemometry (LDA). They were found that the velocity distribution in the impeller stream was very different from the single impeller. 
For the effect of the number of impellers implemented in stirred tank, Franco et al. [7] was found that the effect of the vortex at the impeller blades shows turbulent fluid flow and mixing time shorter than one impeller. In order to carry out an analysis for the stirred tank, there are various technique was used. For example, Zalc et al. [8] was simulated a laminar flow in an impeller stirred tank using CFD tools. They were studied an effect of mixing time performance as a function of the impeller speed. Since the CFD can simulate various configuration of the stirred tank included the impellers and baffles, a lot of analysis can be done numerically.

On the basis of the literature review highlighted here, it can be said that there are a lot of study had been conducted on the mechanical stirred tank, particularly those equipped by various numbers of blades. However, there is lack of some particular system which is discuss regarding the design the blade itself and at the same time the rule of baffles to enhance the mixing process. In this paper, a simulation work has been carried out by using ANSYS Fluent software package to determine the fundamental mechanisms of mixing with a new pattern multi stage stirred tanks with fractal pattern baffled. At the same time, an effect of baffles in the stirred tank on the mixing performance also been discussed. For the simulation work, the authors have focused on the low Reynolds number mixing regime because this situation are the most common problem occurs in practical applications.

Fractal concept. Fractal is basically a repeated shape and self-similarity to an infinitely small scale [9]. According to Karl Weiertrass, the mathematician who was introduced Weierstrass function in 1923, the fractal is continuous everywhere but differential nowhere. Helge Von Koch in 1904 then refined the definition of the Weierstrass function and adds on a more geometric definition that called Koch snowflake. As a high turbulent level occurred when a fluid flow through the fractal pattern [10], we expect that the fractal shape can give a significant effect on mixing effectiveness due to their self similar shape. Another advantages of the fractal pattern that motivated us to apply as a pattern for baffles and impellers in stirred tanks because of low pressure drop across them [11]. Research on the space filling fractal were carried out by Hurst \& Vassilicos [12] who found that the static pressure drop for the space filling fractals is independent of the thickness factor; moreover, away from the grids the homogeneity is improved as a function of the thickness factor. In this research, square grid fractal was chosen as a pattern that will apply to the baffles and impeller for the purpose of research. The square grid fractal is as in Figure 1 below.
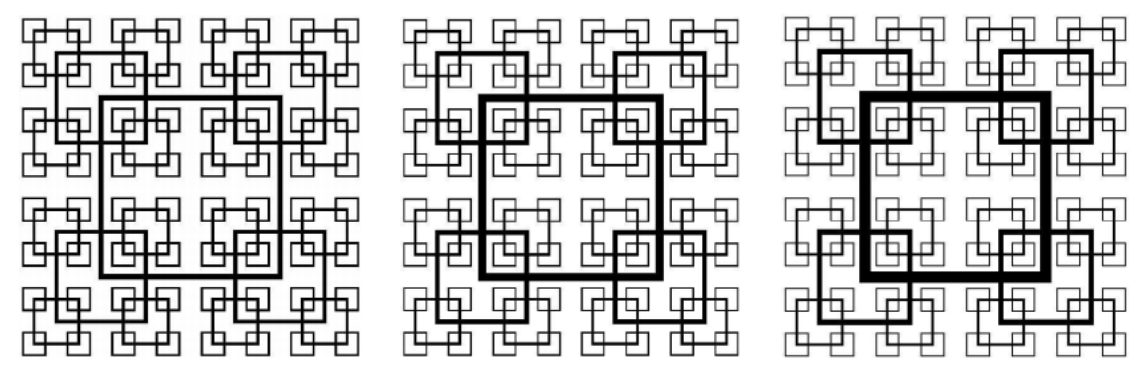

Fig. 1: Square grid space filling fractal with a different thickness ratio [14]

\section{Simulation Work}

For the simulation work, the model of stirred tank was build to investigate flow behaviour in the stirred tank equipped with a fractal impellers and baffles. The model of stirred tank used here is a clear cylindrical tank with a diameter, $T=300 \mathrm{~mm}$. The rest of stirred tank components dimension are referred to the diameter of the tank. This tank was equipped with 4-blade multi-stage impellers with the impeller shaft located at the axis of the tank, a flat bottomed and fitted with four symmetrical baffles at $90 \circ$ interval against the tank wall. The model of stirred tank that was set up for the simulation work is shown in Figure 2 and the dimensions of the tanks are given in Table 1. 


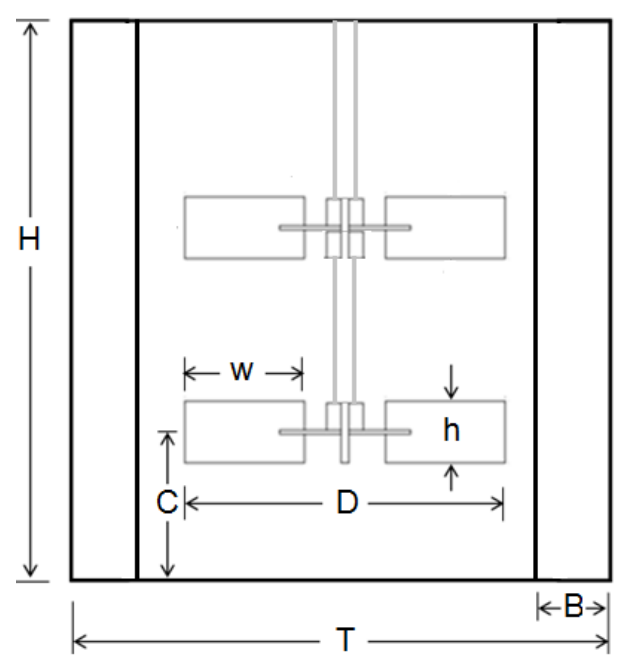

Table 1 Dimensions of the stirred tank designed

\begin{tabular}{lc}
\hline General parameters & $\begin{array}{c}\text { Dimension } \\
(\mathbf{m m})\end{array}$ \\
\hline Tank diameter, $(T)$ & 300 \\
Depth of liquid, $(H)$ & $1 T$ \\
Impeller diameter, $(D)$ & $T / 3$ \\
Impeller blade width, $(w)$ & $D / 4$ \\
Impeller blade height, $(h)$ & $D / 5$ \\
Baffles width, $(B)$ & $T / 10$ \\
Impeller clearance, $(C)$ & $T / 3$ \\
\hline
\end{tabular}

Fig. 2: Schematic diagram of a baffled tank

with a multi-stage turbine impeller.

In this simulation, four configurations of the simulation models were set up and the configurations were chose in order to determine the effect of each configuration for the flow pattern in the stirred tank. The configurations are Standard Baffles and Standard Impellers (SBSI), Fractal Baffles and Standard Impellers $(F B S I)$, Standard Baffles and Fractal Impellers $(S B F I)$ and Fractal Baffles and Fractal Impellers $(F B F I)$. Summary of the configurations are shown in Table 2 below. The simulation model and the meshing were shown in Figure 3 below. In order to investigate the effect of the baffles and impellers configuration, the working fluid used in this simulation was air.
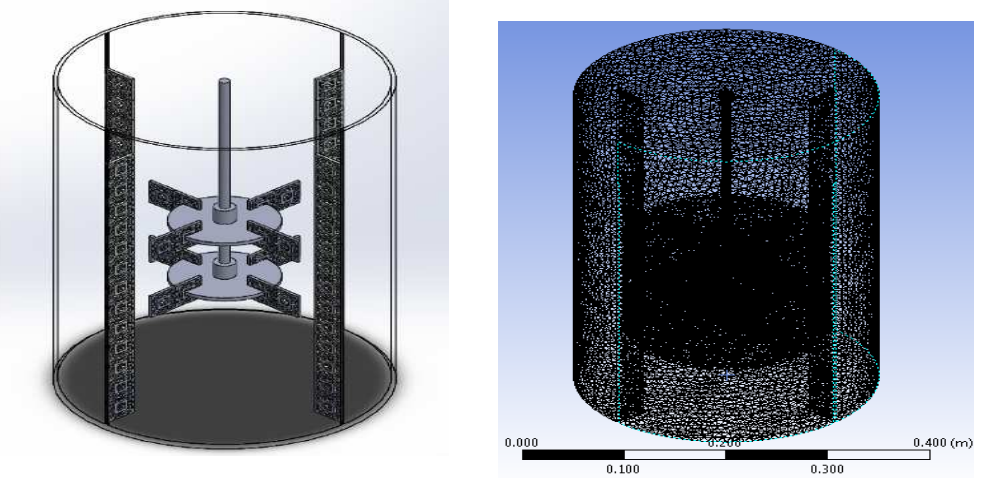

Table 2 Configurations used for simulation work

\begin{tabular}{ccc}
\hline Configuration & Baffles & Impeller \\
\hline $1^{\text {st }}(S B S I)$ & Standard & Standard \\
$2^{\text {nd }}(F B S I)$ & Fractal & Standard \\
$3^{\text {rd }}(S B F I)$ & Standard & Fractal \\
$4^{\text {th }}(F B F I)$ & Fractal & Fractal \\
\hline
\end{tabular}

Fig. 3: Simulation model for the stirred tank with fractal baffles and impellers and the meshing of the model

\section{Results and Discussion}

The results for velocity distribution and velocity vector were presented by velocity contour as in Figure 4. In the figures, it is clearly shows a significant effect of the fractal pattern in the stirred tank in term of velocity distribution. The first image represents the first configuration which is standard baffles and standard impeller. Basically this is a reference and common flow occurred in current stirred tank available nowadays. For this combination, the flow distribution are mostly scattered in all region around the tank and a little bit small vortex occur between the impellers. The fluid also concentrated at the top of the moving impeller. This condition will cause a high pressure drop and hence increase the power consumption for the mixing process. 


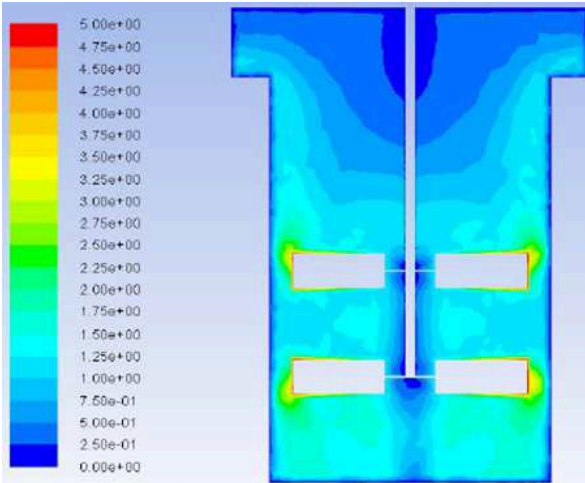

SBSI
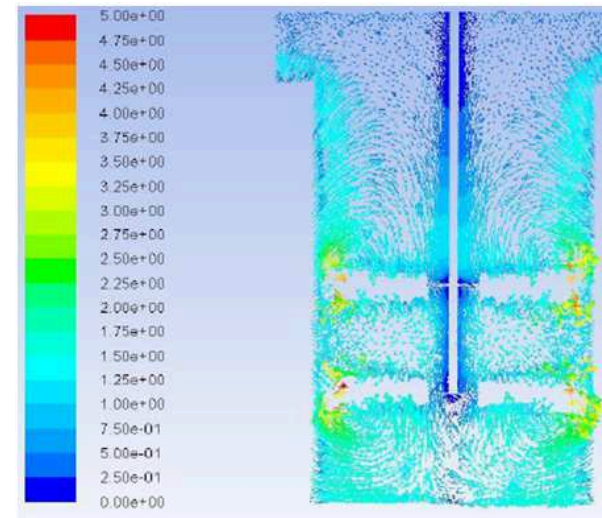

SBSI

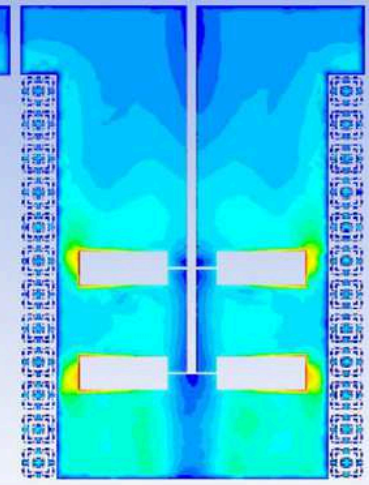

FBSI

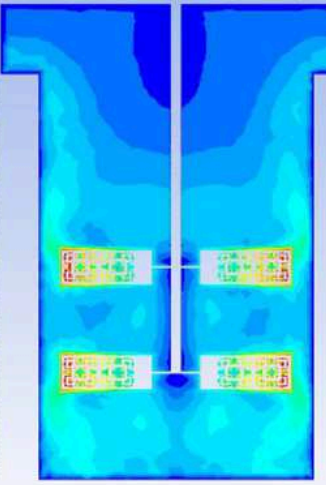

SBFI

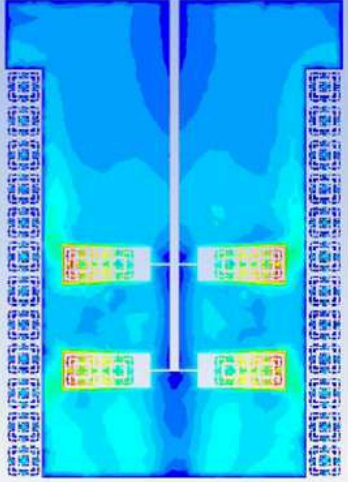

FBFI

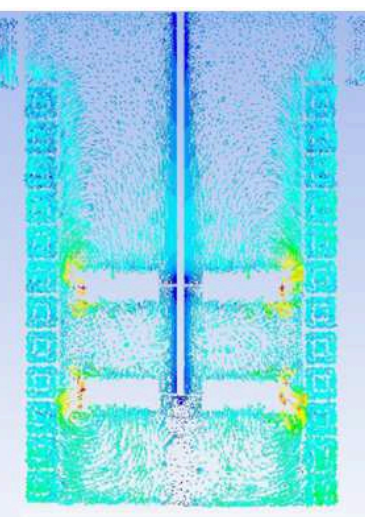

FBSI

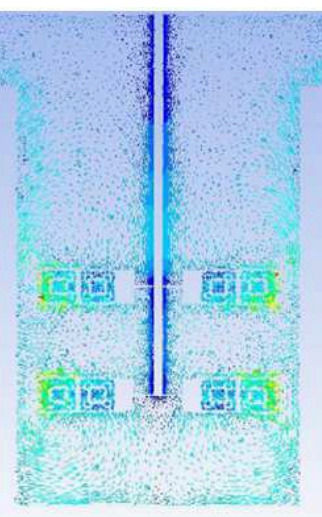

SBFI

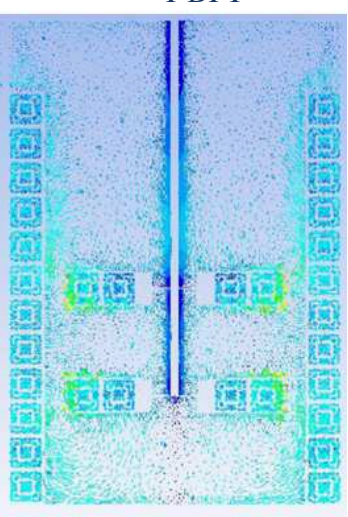

FBFI

Fig. 4: Velocity contour and velocity vector for four configurations of simulation models

Second configuration which is FBSI, it showed that the flow have a nearly similar pattern as in the first configuration. However the flow moved smoothly around the baffles and this give advantages due to low pressure drop when the fractal baffles implemented to the stirred tank. Again for the implementation of fractal baffles for the third configuration, SBFI, the flow pattern are clearly similar when it cross the fractal impellers and the flow around the impellers had a good movement without any concentrated particles around that. The last configuration which is the implementation of fractal pattern for both baffles and impellers give the best configuration in this study. The results showed in the last image in Figure 4 above gave a clear view on how the fractal pattern influenced the flow in the stirred tank. The fluid particle seems to be fairly distributed in the tank and there is no fluid concentration on the fractal baffle as compared to normal baffle on other configurations. The results gave a good agreement with a previous research which is the fractal pattern can generate high turbulent level; hence enhance the mixing in the stirred tank.

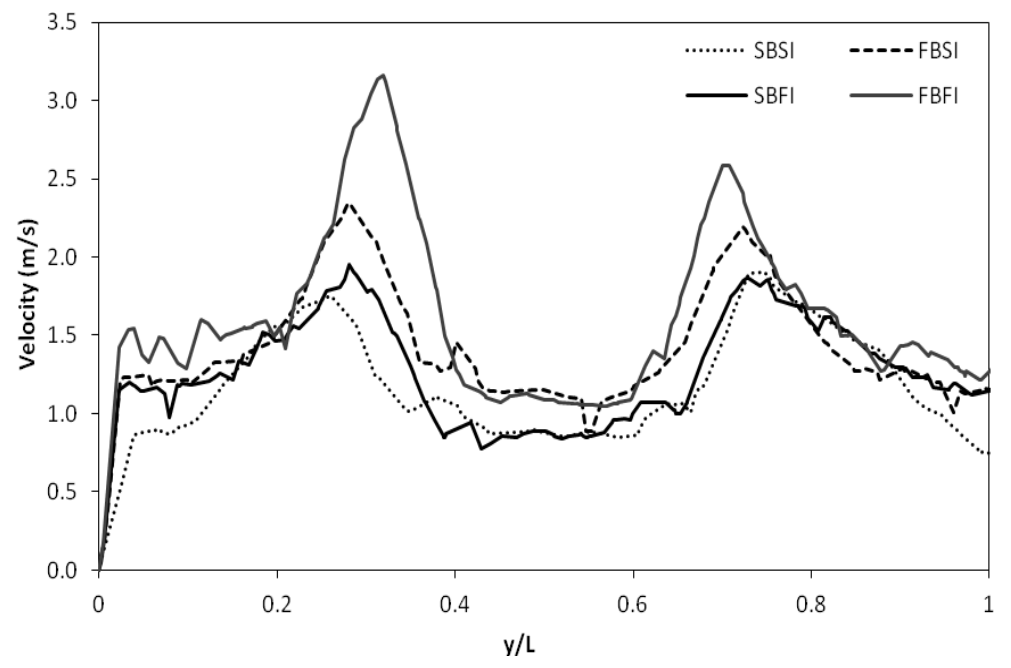

Fig. 5: Velocity distribution along the vertical line at distance T/4 from centre of stirred tank 
Besides the flow pattern that clearly gave a good result in term of the implementation of fractal baffles and impellers for a stirred tank, the velocity plot along a selected vertical location in the stirred tank also were come out as a result in this paper. Figure 5 showed a velocity distribution along the vertical line at distance $\mathrm{T} / 4$ from the centre of stirred tank. As we can see from the figure, high velocity occurred at the area near the impeller tips. More interesting here is the highest velocity occurred at the fractal impeller where it is important for a fluid mixing to have a high velocity to ensure a homogeneous mixing in the tank.

\section{Conclusion}

As a conclusion, this research study of the new approaches of fractal baffles and impellers have successfully done by fulfilling the requirement of main objectives for this study. The concept of the square grid was developed based on fractal pattern and has been applied to generate new approach of baffles and impellers in stirred tank. The simulation results were showed that the velocity contour and velocity magnitude have a different flow pattern in stirred tank by using fractal design on four blade multi-stage impellers and baffles. Besides that, the highest velocity occurred at the fractal impellers tips and it gave advantages of the fractal designed impeller where the highest velocity can give an influence in mixing criteria in stirred tank. Although this idea is a basic concept, it can be improved in order to provide better results in term of fluid mixing and also the flow pattern in stirred tank.

\section{Acknowledgement}

Partial support was provided by MOHE (Ministry of Higher Education, MALAYSIA) and Malaysian Technical University Network (MTUN) under MTUN-CoE Research Grant Scheme, vote $\mathrm{C} 010$.

\section{References}

[1] Zadghaffari R, Moghaddas J.S. and Revstedt J. A Study on Liquid-Liquid Mixing in a Stirred Tank with a 6-Blade Rushton Turbine, Iranian Journal of Chemical Engineering. (2008) 5(4): 12-22.

[2] Aubin J., Kresta S.M., Bertrand J., and Fletcher D.F. Alternate Operating Methods for Improving the Performance of a Continuous Stirred Tank Reactor, Trans IChemE, (2006) 84:569-582.

[3] Zadghaffari R., Moghaddas J.S., and Revstedt J. A Study on Liquid-Liquid Mixing in a Stirred Tank with a 6-Blade Rushton Turbine, (2008) 5(4):12-22

[4] Kchaou H., Driss Z., Bouzgarrou G., Chtourou W., and Abid M.S. Numerical investigation of internal turbulent flow generated by a flat-blade turbine and a pitched-blade turbine in a vessel tank, International Review of Mechanical Engineering. (2008) 2:427-434.

[5] Driss Z., Bouzgarrou G., Chtourou W., Kchaou H., and Abid M.S. Computational studies of the pitched blade turbines design effect on the stirred tank flow characteristics, European Journal of Mechanics B/Fluids. (2010) 29:236-245.

[6] Mishra V.P. and Joshi J.B. Flow generated by a disc turbine multiple impellers, Trans. IchemE, 7(1994) 2:657-668

[7] Franco M., Giuseppina M., Davide P., and Alessandra P, Mixing time in high aspect ratio vessel with multiple impeller, Chemical Engineering Science, (2013) 101:712-720

[8] Zalc J.M., Szalai E.S., Alvarez M.M. and Muzzio F.J., Using CFD to understand chaotic mixing in laminar stirred tanks, AIChE Journal, (2002), 48;2124-2134.

[9] Manshoor B., Zaman I., Jaat M., and Amir Khalid. CFD Analysis of Circle Grid Fractal Plate Thickness on Turbulent Swirling Flow, Applied Mechanics and Materials. (2014) 466: 109-113 
[10] Manshoor B, Nicolleau FCGA and Beck SBM. The fractal flow conditioner for orifice plate flow meters. Flow Measurement and Instrumentation. (2011) 22:208-214

[11] Manshoor B, Zaman I. and Amir Khalid. Experimental study of pressure drop after the circle grids fractal orifice. Applied Mechanics and Materials. (2010) 390:111-115

[12] Hurst D. and Vassilicos J.C., Scalings and decay of fractal-generated turbulence. Physics of Fluids. (2007) 19(3). 